\title{
Experiencia de la clase inversa en didáctica de las lenguas extranjeras
}

\section{A flipped-classroom experience in Didactics of Foreign Language}

\author{
María Asunción Barreras Gómez ${ }^{1}$ \\ asuncion.barreras@unirioja.es \\ Universidad de La Rioja, España
}

\section{Resumen:}

Este artículo se centra en la aplicación del modelo de la clase inversa en la asignatura de didáctica de las lenguas extranjeras en la Universidad de La Rioja. Esta metodología invierte los modelos tradicionales en el proceso de enseñanza-aprendizaje, facilitando pautas de trabajo online desde fuera de la clase. En el aula el profesor ayuda al alumno en la aplicación de esos contenidos y se puede centrar en las necesidades del alumno. Para ello se han utilizado varias herramientas como Power Points, Youtube, aunque se destaca especialmente Wikis y Kahoot. Cuando se planteó esta metodología los alumnos parecieron un poco desorientados. Sin embargo, la experiencia ha resultado ser muy beneficiosa.

Palabras claves:

Clase inversa; Wiki; Kahoot.

\begin{abstract}
:
This article is based on the application of the flipped classroom model to the subject Didactics of Foreign Language at the University of La Rioja. This methodology inverts the traditional models in the teaching-learning process, promoting online homework to be completed outside the class. Once in the classroom, the teacher helps students apply theoretical contents to practical cases. Consequently, the teacher can focus on the students' weaknesses. In so doing, technical tools such as Power Points, Youtube and especially Wikis and Kahoot have been adapted. At the beginning of the experience the students were disoriented. However, it proved to be successful in the end.
\end{abstract}

\section{Key words:}

Flipped Classroom; Wiki; Kahoot.

1 Dirección para correspondencia (correspondence address):

$M^{a}$ Asunción Barreras Gómez. Dpto. de Filologías Modernas. Facultad de Letras y de la Educación. Universidad de La Rioja. Edificio de Filologías. San José de Calasanz, s/n. 26004 Logroño (España). 


\section{Résumé:}

Cet article présente l'application du modèle de la classe inversée dans la matière de didactique des langues étrangères à I'Université de La Rioja. Cette méthode inverse les modèles traditionnels du processus d'enseignement/apprentissage, en favorisant le travail en ligne à la maison. Cela permet au professeur d'aider l'étudiant dans la mise en pratique des contenus et en même temps de se centrer sur les besoins de l'élève. Pour ce faire, nous avons employé plusieurs outils informatiques tels que des présentations powerpoint, des clips de Youtube, mais surtout des Wikis et la plateforme kahoot. Au début de cette expérience méthodologique, les étudiants semblaient un peu désorientés. Pourtant, l'expérience s'est avérée vraiment profitable.

\section{Mots clés:}

Classe inversée; Wiki; Kahoot.

Fecha de recepción: 9-1-2015

Fecha de aceptación: 17-1-2016

A Isabel Gómez Alemany, Profesora de la Universidad Autónoma de Barcelona, in memoriam

\section{Introducción}

Con la repercusión que la sociedad de la información y las nuevas tecnologías tienen en todos los niveles del mundo educativo, muchos docentes se están replanteando los contenidos del currículum así como las metodologías que utilizan en el aula. Al explicar las habilidades del siglo XXI necesarias para el aprendizaje, Trilling y Fadel (2009: 48-49) destacan cuatro destrezas de aprendizaje crítico e innovación: creatividad, pensamiento crítico, comunicación y colaboración, ya que consideran que son básicas en el aprendizaje y el trabajo creativo. También destacan como tópicos básicos del siglo XXI las habilidades profesionales y para la vida, las destrezas de aprendizaje e innovación así como las de la información, medios y tecnología. Estos aspectos se señalan específicamente para la universidad en la declaración de Bolonia: "la importancia de focalizar la formación en el aprendizaje del alumnado como sujeto activo a través del desarrollo de un modelo de enseñanza basado en el diseño de competencias" para ayudarles en su futuro profesional y "la incorporación de modelos de formación orientados hacia el dominio de la competencia tecnológica" (Ricoy y otros 2011, p. 485). Se deja atrás 
la figura del magister dixit de antaño. El profesor ya no es un dogmático y no necesariamente tiene un papel protagonista en la clase. Las clases se pueden centrar en los alumnos, el papel del profesor pasa a ser el de un colaborador dialogante y la tecnología tiene un papel relevante en este cambio.

Con este contexto la convergencia europea se plantea como una oportunidad para mejorar la docencia universitaria. En este reto Zabalza (2006: 48-54) resalta que la docencia se centre en el estudiante, su aprendizaje autónomo y la utilización de las TICs en este nuevo contexto. El aprendizaje autónomo permite dejarles tiempo a los alumnos para trabajar solos o en grupo y supone también un protagonismo de las tecnologías para que las puedan utilizar en su proceso de aprendizaje y a su ritmo. De Miguel (2006: 75-76) destaca en este sentido que el docente tendrá que proporcionar al alumno las herramientas para llevar a cabo "su búsqueda personal hacia el aprendizaje" y que las tecnologías de la información son fundamentales para "proceder a esta renovación de metodológica". El profesor no solamente va a ofrecer conocimientos, además les enseñará "pautas, herramientas y medios para que sean los propios alumnos" los que "desarrollen su propio proceso de aprendizaje". De esta manera, el profesor les orientará y guiará en el proceso cognitivo de la construcción de su propio conocimiento.

Muchos profesores universitarios han empezado a integrar recursos de la Web $2.0^{2}$ para su docencia. En la Universidad de La Rioja se está trabajando en un proyecto de innovación denominado "Estudiantes móviles y aprendizaje en Educación Superior: Adaptación del enfoque Flipped classroom en un contexto Boyd". El objetivo es integrar curricularmente los recursos tecnológicos y para ello se está adaptando el enfoque Flipped classroom con las tecnologías móviles.

\section{The flipped classroom o clase inversa}

Esta metodología invierte los modelos tradicionales en el proceso de enseñanza-aprendizaje, facilitando pautas de trabajo online desde fuera de

2 Se trata de un término que cubre todas las aplicaciones de la web para permitir a los usuarios crear y compartir información así como colaborar con la web. Los servicios de segunda generación basados en la web incluyen herramientas que permiten la colaboración y compartir la información online (Spector y otros 2014: 964). 
la clase y trasladando determinadas tareas dentro de la clase. El método tradicional representa al docente como la persona que imparte la clase y "deberes" para el día siguiente. En este modelo el profesor permanece a un lado ejerciendo de guía, mientras que los estudiantes trabajan en la clase. El modelo requiere que los alumnos realicen determinadas actividades como ver videos online en casa, en su propio espacio, con la posibilidad de comunicarse con otros alumnos y el profesor mediante debates online. Esta idea ha ido tomando relevancia a medida que se iban desarrollando la tecnología y su acceso a los estudiantes. De acuerdo con Randall, Dean y Ball (2013: 565), los alumnos aprenden conceptos, hacen actividades a su ritmo para poder tratarlo en profundidad en clase y es en el aula donde los conceptos se afianzan con la ayuda del profesor. Éste puede adaptar las sesiones basándose en los resultados de las tareas que los alumnos han hecho en clase o en los debates que se den en la misma. De esta manera el profesor es consciente de los problemas que se encuentran los alumnos de forma individualizada y les puede ayudar. Además el docente les ofrece recursos con los que los alumnos vayan construyendo su propio aprendizaje de forma autónoma con ejemplos online tanto escritos como visuales. Así los alumnos pasan a tener un papel activo (Zuraidah 2014: 126) en su aprendizaje. La tecnología ayuda en ese proceso. Los tradicionales deberes y el tiempo de clase se intercambian de lugar. El contenido se relaciona más con escenarios reales y la clase se utiliza para un pensamiento crítico y resolución de problemas.

Como se puede apreciar en The Flipped Classroom, la tecnología y las actividades de aprendizaje son básicas. Ambas influyen en el entorno de aprendizaje del estudiante de manera fundamental. Es interesante que el profesor tenga una cierta competencia digital para poder mostrar a los alumnos herramientas tecnológicas que les sean útiles así como fáciles de utilizar.

En la lista de herramientas de aprendizaje más útiles del Centre for Learning \& Performance Technologies (C4LPT ${ }^{3}$ ) en el año 2014 aparecen en las veinte primeras posiciones herramientas como: Twitter, Google Docs/Drive, YouTube, Power Point, Google Search, WordPress, Dropbox, Evernote, Facebook, LinkedIn, Google+\& Hangouts, Moodle, Prezi, Pinterest, Slideshare, Blogger, Word, Wikipedia, Freedly y Digo.

3 Es la página web más visitadas sobre información relativa a las tendencias, tecnologías y herramientas de aprendizaje. Véase http://c4lpt.co.uk/ 
El radiocasete o el vídeo, por ejemplo, han sido materiales bastante usados en el aula en los últimos veinte años. Además, el vídeo es un formato de gran impacto y repercusión actualmente en el desarrollo de sitios 2.0. Entre los servidores de video, destaca Youtube por su volumen y relevancia social. Algunas estadísticas avalan esta afirmación: Cada día más de 2000 millones de videos son visualizados en Youtube. Este avance tecnológico tiene que tener su repercusión en todos los aspectos, incluido el educativo. En concreto, con herramientas como las mencionadas en las lista de Centre for Learning \& Performance Technologies, Walld y otros (2014: 1) argumentan que los alumnos pueden trabajar en sus casas a su ritmo, haciendo descansos, visionando videos con el tamaño requerido y el volumen preferido, viéndolos cuantas veces quieran y tomando las notas que necesiten así como compartiendo sus ideas; por lo que se facilita el aprendizaje autónomo.

Para que este tipo de aprendizaje autónomo sea posible tiene que tener un apoyo decidido en el centro de educación. Walling (2014: 77) destaca que debe haber un ambiente flexible que involucre a los alumnos y una cultura proclive al cambio de una clase centrada en el profesor a una centrada en el alumno. También añade que es relevante trabajar con estrategias activas de aprendizaje durante la clase.

Estos serán algunos de los aspectos que se pretenderán desarrollar con esta metodología en la asignatura de didáctica de las lenguas extranjeras del grado de Educación Primaria para llevarla a cabo en la Universidad de La Rioja.

\section{Contexto}

Como se ha mencionado anteriormente, en la Universidad de La Rioja se está trabajando en un proyecto de innovación cuyo objetivo es integrar curricularmente los recursos tecnológicos, adaptando el enfoque Flipped classroom con las tecnologías móviles. El contexto concreto de este proyecto es el de la asignatura de la didáctica de las lenguas extranjeras. Se trata de una asignatura optativa que cursan los alumnos para conseguir la mención de lengua inglesa en su Grado de Educación Primaria, por lo que se enfoca hacia la enseñanza de la lengua inglesa. Esta asignatura se cursa en el tercer curso de dicho grado. Por tanto, los alumnos ya han estudiado materias relacionadas con el aprendizaje y 
desarrollo de la personalidad, procesos y contextos educativos, sociedad, familia y escuela, enseñanza y aprendizaje de las lenguas así como enseñanza y aprendizaje de la educación musical, plástica y visual. Esto les ha dado una buena base para entender mejor la enseñanza del inglés en un aula de Educación Primaria. Por otro lado, tienen un amplio conocimiento de la lengua inglesa por las asignaturas específicas de inglés estudiadas para poder interaccionar en inglés. Además, estos estudiantes son digitalmente competentes, ya que normalmente traen sus dispositivos móviles, bien para acceder a los servicios de la Universidad de La Rioja o para trabajar en las aulas con sus compañeros y profesores. Así, este proyecto se enmarca en un contexto BYOD (Bring Your Own Device, en castellano "trae tu propio dispositivo").

Los alumnos están acostumbrados a utilizar sus dispositivos electrónicos de forma eficaz para acceder a diversos recursos y conseguir información útil en el contexto docente. Como destacan Pilgrim, Bledsoe and Reily (2012: 16), en los centros educativos los alumnos encuentran acceso a correos electrónicos, bases de datos, diccionarios electrónicos y archivos en servidores así como aplicaciones personales. En este sentido, las instituciones educativas deben dar servicio a un gran número de estudiantes que están en constante contacto con la tecnológica, puesto que cada vez es más frecuente encontrar a alumnos utilizando su propio teléfono móvil o su propia tableta para acceder a la red universitaria. Este es el caso de la Universidad de La Rioja.

Walling (2014: 4) resalta la accesibilidad a la información que ofrece una tableta. También la posibilidad de aprendizaje colectivo que ofrece más allá del aula y el acceso a todos los libros de texto con un mismo dispositivo móvil así. Además, al profesor le facilita la posibilidad de evaluación de los alumnos.

Este autor (Walling 2014: 107) también subraya algunos retos que tiene el profesor al llevar a clase la estrategia BOYD. El primero es la instrucción individualizada dentro de los límites del dispositivo móvil con el que cuente el alumno. Los dos siguientes consisten en conseguir un software disponible o localizar uno cuando no lo haya. El cuarto es utilizar una instrucción de grupo grande u otras formas de agrupamiento, de tal forma que se puedan evitar posibles problemas tecnológicos. El último es el agrupamiento de estudiantes de acuerdo con sus posibilidades de utilización de dispositivos móviles, más que por otras características. Para ello la Universidad de La Rioja cuenta con una plataforma Black- 
board. Se trata de un software que permite realizar actividades como cursos, subir materiales didácticos, comunicarse con los alumnos por medio de foros de discusión o Chat, hacer seguimiento de su proceso de aprendizaje e, incluso, calificar a los alumnos con las rubricas o el panel de control de evaluación.

\section{Objetivos}

El modelo de la clase inversa en la asignatura de didáctica de las lenguas extranjeras, junto con la tecnología de la que disponen los alumnos y la de la universidad de La Rioja, permitirá a éstos desarrollar las siguientes competencias requeridas para su futuro profesional:

- Creatividad e innovación.

- Pensamiento crítico y resolución de problemas

- Comunicación y Colaboración

- Habilidades para la vida y el trabajo

- Desarrollo de la competencia tecnológica

Como veremos a continuación, el uso del aula virtual por parte de los alumnos es importante en este contexto digital para que puedan obtener estos objetivos finales. Es capital desarrollar el aula virtual de manera que los alumnos puedan interactuar autónomamente y que puedan acceder a distintos recursos de información. Para ello se ha distribuido la información en diversas carpetas; en este sentido cabe destacar la carpeta Textos prácticas, que es la más reciente y cuyo objetivo es que los alumnos utilicen la $W_{i k i^{4}}$ de la asignatura para poder crear debates de grupo, un proyecto en grupo o crear un diccionario en la clase.

Finalmente, hay que mencionar que el objetivo de este artículo es mostrar un ejemplo de la aplicación de la metodología de la clase inversa aplicada a la asignatura de didáctica de las lenguas extranjeras y comprobar hasta qué punto los alumnos desarrollan las competencias referidas.

4 Procede de la palabra Hawaiana rápido. Posiblemente sea esa la característica relevante de esta herramienta, ya que un grupo de usuarios pueden crear, cambiar o borrar sus contenidos de forma interactiva, rápida y fácil (Rodríguez-Arancón y Calle Martínez 2014: 159). En la lista de herramientas de aprendizaje más útiles del Centre for Learning \& Performance Technologies (C4LPT) en el año 2014 aparece la herramienta de wikispaces en la posición octogésimo cuarta y la Wikipedia, en la decimoctava. 


\section{Experiencia de "The Flipped Classroom" en la asignatura de didáctica de las lenguas extranjeras.}

En la asignatura de didáctica de las lenguas extranjeras se ha utilizado la metodología The Flipped Classroom por primera vez durante el curso 2013-2014. Por esta razón y como ya se ha mencionado, la utilización del aula virtual de la asignatura tenía que incrementarse. Para ello se ha ampliado la información a la que podían acceder los estudiantes, plantear distintas interacciones y mejorar la estructura del aula para facilitar a los alumnos la ubicación de los distintos materiales.

Desde principio de curso esta asignatura ha contado en su aula virtual con varias carpetas de interés para los alumnos. De esta manera se cumple con uno de los puntos de interés de la metodología inversa, ofrecer a los alumnos recursos para usar fuera de la clase de forma que el tiempo de clase se dedique a actividades de instrucción (Enfield 2013: 14). Estas carpetas que se comentan a continuación las tienen los alumnos en el aula virtual de la asignatura.

- Información general: Guía y cronograma de la asignatura.

- PPts: los power points de las unidades didácticas.

- Articles: Artículos y capítulos de libros en los que se basan las unidades didácticas de la asignatura y que los alumnos deben leer antes de ir a clase.

- Textos prácticas: Artículos y capítulos de libros que los alumnos deben resumir o esquematizar para colgarlos en una de las Wikis de la asignatura.

- Textos audiovisuales: Textos audiovisuales que los alumnos pueden encontrar en la red y que se utilizarán en las clases de grupo grande o de grupo reducido y que, además, se podrán comentar en una de las Wikis de la asignatura.

- Textos escritos: Artículos y monografías que los alumnos pueden encontrar en la red y que se les facilita el acceso con el enlace directo.

- A modo de ejemplo: Textos escritos que ejemplifican distintos aspectos prácticos de la asignatura.

- Curiosidades: Textos escritos y visuales de diversos personajes ficticios o reales que comentan algo relacionado con la asignatura. 
Se les ofrece un tipo de instrucción multimedia que incluye textos escritos y orales junto con ilustraciones, diagramas, videos o fotografías. Una de las razones que también justifican su utilización es que se puede aprender más con palabras e imágenes que solamente con palabras, lo que se ha denominado el principio multimedia (Mayer 2014: 385). Con esta información los alumnos han tenido que utilizar herramientas de las tecnologías de la información y la comunicación en la asignatura de didáctica de las lenguas extranjeras. En la experiencia de clase inversa en esta asignatura durante el curso 2013-14 se han utilizado principalmente dos herramientas de la lista del Centre for Learning \& Performance Technologies, Wikis y Kahoot, aunque también se han utilizado otras herramientas como YouTube, Power Point o Google Search en el proceso de aprendizaje de los alumnos.

\subsection{Wikis en la didáctica de las lenguas extranjeras}

Walling (2014: 111) destaca que muchos estudiantes aprenden de manera más efectiva en comunidad y crean comunidades de aprendizaje. Sobre este punto Ibáñez y Gómez Alemany (2004: 69) añaden que la gestión cooperativa del aula favorece la autorregulación del aprendizaje, la autogestión de errores, promueve la discusión, el desarrollo de estrategias y la toma de decisiones. En este sentido, la Wiki ayuda en las tareas colaborativas de los alumnos. Hsu y otros (2014: 751) explican que las Wikis capacitan a los usuarios a editar el trabajo de otros en la misma página, monitorizar la revisión hecha por otros usuarios y retrotraerse a versiones anteriores. En consecuencia, al final, las Wikis se convierten en artefactos de construcción colectiva del conocimiento. Así los alumnos desarrollan el pensamiento crítico en un espacio compartido. Cabe destacar que en la experiencia con la asignatura de didáctica de las lenguas extranjeras los alumnos aprendieron a manejar la Wiki. Una vez en la página de herramientas del aula virtual de la asignatura, fácilmente se introducían en la Wiki y podían introducir sus aportaciones o comentar las de otros compañeros. Para cada unidad didáctica los alumnos se distribuían por grupos para leer artículos o visionar un video relacionado con el tema objeto de estudio, bien en castellano o bien en inglés, para resumirlo y comentarlo. Si el texto era demasiado largo o los alumnos de un grupo tenían dificultad en poder quedar, éstos utilizaban Google docs ${ }^{5}$

5 En la lista de herramientas de aprendizaje más útiles del Centre for Learning \& Performance Technologies (C4LPT) en el año 2014 aparece la herramienta de documentos 
para redactar el resumen y comentario del mismo de manera previa. Después tenían que subir a la Wiki de ese tema los resúmenes y comentarios hechos, explicando las ideas esenciales, y, para ello, podían utilizar la forma narrativa con un resumen o presentar un esquema. Cada Wiki ha contado con varias entradas, las del resumen y reflexión personal de cada de cada texto. En cada una de estas entradas el resto de los compañeros podían comentar o añadir ideas e información relacionada, por lo que algunas Wikis se alargaban más que otras.

Los temas que se han tratado en las Wikis tienen que ver con la enseñanza del inglés en primaria: Tipos de actividades que pueden involucrar mejor a estudiantes tan pequeños, utilizar un libro de texto a no utilizarlo en clase, las destrezas orales y escritas del inglés como lengua de aprendizaje, los textos literarios infantiles en la enseñanza del inglés o diversos tipos de metodologías de enseñanza de idiomas, como Total Physical Response, CLIL (Content and Language Integrated Learning) o Jolly Phonics, etc. En las Wikis los alumnos han tenido que trabajar esos temas de una manera más teórica, en el sentido que han tenido que resaltar las ideas más importantes y reflexionar sobre ellas. En las clases se comentaban las dudas que pudieran surgir, se comparaban y relacionaban conceptos de los textos. Se traían libros de textos de primaria para analizarlos y comentar sus características entre todos y sugerir posibles actividades alternativas o de refuerzo para los temas del libro de texto. Si se trataba de videos donde algún profesor estuviera dando una clase en un aula de primaria (por ejemplo de Total Physical Response, CLIL o actividades llevadas a cabo con niños de primaria), también se comentaban las técnicas que utilizaba para captar la atención de los alumnos de primaria, los materiales que utilizaba, la manera de utilizar el lenguaje corporal para dar más significado a lo que estuviera diciendo en inglés, etc. Incluso los propios alumnos universitarios tenían que contar un cuento en inglés y diseñar actividades, relacionadas con las cuatro destrezas (listening, speaking, reading y writing), para niños de primaria; de tal forma que pusieran en práctica aspectos que se de manera teórica se comentaban en clase.

Como los textos de lectura los habían trabajado todos los alumnos,

de Google (google docs) en cuarta posición. Esta herramienta permite trabajar en un mismo documento a varios usuarios, posibilitando modificaciones en tiempo real así como chateando y comentando sobre el texto que están escribiendo. Esta herramienta convierte archivos de Word en documentos Google y viceversa para luego poderlos copiar, por ejemplo, en la Wiki de esta asignatura. 
en clase todos ellos participaban en mayor o menor medida. Para ello se había creado un ambiente distendido en clase, en el que todos se sintieran cómodos participando. Se destaca la flexibilidad, junto con el hecho de que los propios alumnos podían elegir cuándo tomar la palabra y hablar delante de todos. Además, esta herramienta provocó que, al tener que realizar esquemas, los alumnos fácilmente utilizaran Mind mapping y Popplet $t^{6}$ también para organizar la información visualmente. De acuerdo con Novak y Cañas (2006: 1), son herramientas gráficas para organizar y representar el conocimiento. Incluyen conceptos en círculos o cajas, las relaciones entre los conceptos, indicando la unión entre dos conceptos, donde las palabras sobre las líneas especifican la relación entre dos conceptos. Además con Mind mapping y Popplet los alumnos podían utilizar herramientas que le servían para aprender a la vez que lo podían aplicar al mundo real (Pilgrim, Bledsoe and Reily 2012: 21), ya que lo podrían utilizar también en su futura docencia para explicar visualmente un concepto a sus alumnos en la asignatura del Prácticum y en su futuro profesional.

Lott (2005) comenta que las Wikis en educación sirven como un espacio de comunicación en el aula. La facilidad con la que se crea y edita el contenido en una Wiki contrasta con el tipo de comunicación jerarquizada y estructurada de otras herramientas como los foros. Por eso, puede ser un lugar de conocimiento colaborativo sobre un tema específico, donde aparezca la labor de investigación de los alumnos. Añade que la Wiki puede ser, además, un lugar donde los alumnos puedan mostrar sus trabajos para que el profesor lo pueda evaluar. Pero, subraya que por la flexibilidad que presenta esta herramienta para crear y editar un texto, puede ser el lugar perfecto para crear un texto de forma colaborativa, aunque los alumnos se encuentren físicamente lejos. El autor también comenta que puede dar lugar a la creación de libros de texto, colección de problemas, manuales, etc. Finaliza diciendo que una Wiki puede ayudar a un grupo de alumnos a publicar su proyecto así como a la creación de actividades destinadas a la autoevaluación o la evaluación de sus compañeros.

En el caso concreto de la asignatura de didáctica de las lenguas extranjeras, la Wiki se ha utilizado por grupos para trabajar sobre temas

6 Se crean mapas conceptuales sin dibujarlos. Presentan los conceptos de manera visual y esquemática. Los documentos se crean y se comparten fácilmente. Véase http:// www.mindmapping.com/ y http://popplet.com/ 
variados, como métodos de enseñanza del inglés, las destrezas orales y escritas del inglés o las implicaciones de las características de los niños para la enseñanza del inglés. Cada grupo ha tenido que trabajar sobre un tema para el que tenían que leer artículos, reflexionar sobre los mismos en las Wikis. Dependiendo del momento de trabajo, no todos los alumnos se encontraban físicamente juntos ni había un momento específico en las clases para trabajar en la Wiki, por lo que esta herramienta, como la de Google docs con los miembros de cada grupo al preparar su resumen y comentarios, ha salvado la distancia y ha facilitado el trabajo de los distintos grupos, haciéndolo más colaborativo entre todos los alumnos de la clase. Luego cada grupo comentaba el trabajo de los otros y todos adquirían una visión más amplia de la asignatura, ya que no solamente se centraban en su tema sino que conocían y aprendían del de los demás grupos. De esta forma, se ha llegado a convertir en "una enciclopedia virtual con contenidos claves para el curso" (Breeze 2014: 113). Participaron todos y esta herramienta les involucró especialmente en la asignatura.

\subsection{Kahoot en la didáctica de las lenguas extranjeras}

Esta relación con las nuevas tecnologías llevó a utilizar el sistema de respuesta en el aula basado en el juego denominado Kahoot ${ }^{7}$. Para su utilización en el aula se requiere un dispositivo móvil, que prácticamente todos los alumnos de la asignatura de didáctica de las lenguas extranjeras tenían; esencialmente disponían de Smart Phone con Windows, a pesar de tener que utilizar una pantalla pequeña, aunque también utilizaban tableta con Android y, en menor medida, Blackberry-RIM.

Icard (2014: 40) comenta que en el aprendizaje basado en la gamificación ${ }^{8}$ los juegos digitales crean una involucración activa en el usuario. Están diseñados para integrar contenidos de aprendizaje con la idea de jugar; lo que permite al cerebro procesar la información de la memoria a corto plazo a la memoria a largo plazo. Crean una implicación activa en

7 En la lista de herramientas de aprendizaje más útiles del Centre for Learning \& Performance Technologies (C4LPT) en el año 2014 aparece la herramienta Kahoot como nueva y en la posición octogésimo primera.

8 Es un anglicismo procedente de las palabras inglesas game y gamification. Trata de la utilización del juego y sus estrategias (sistema de puntos para conseguir un puesto, por ejemplo) en contextos que no son especialmente lúdicos para involucrar, motivar, concentrar y conseguir que los usuarios se esfuercen más. 
los estudiantes que les hace desarrollar las destrezas de la resolución de problemas en ambientes de aprendizaje. Establecen un ambiente lúdico que permite a los alumnos aprender de sus errores y añade que estas simulaciones pueden ayudar a los alumnos a tratar el error en situaciones reales. Finalmente, el autor acaba ejemplificando este tipo de juegos con el reciente diseño de juego Kahoot, que también se ha utilizado en esta experiencia de clase inversa con la asignatura de la didáctica de las lenguas extranjeras.

Para jugar los alumnos se introducen en https://Kahoot.it con un nombre de usuario a un aula virtual creada por el docente o por uno de sus compañeros. Una vez que todos los alumnos se han dado de alta, el juego comienza. Cada uno conseguirá los puntos dependiendo de las respuestas correctas y del tiempo invertido en las contestaciones, puesto que previamente los segundos para responder cada pregunta han sido delimitados por el que ha escrito las preguntas con sus posibles respuestas. Al final aparecen los puntos obtenidos y la clasificación obtenida.

Con este sistema de respuestas, Kahoot, se pueden preparar actividades en la web de precalentamiento para un tema o actividades de enseñanza y revisión de una unidad didáctica. Este sistema, como aparece en su página web, invierte la clase, maximiza el tiempo de aprendizaje utilizado en el aula y permite al alumno dirigir la interacción en el aula. Este sistema favorece la investigación independiente y colaborativa y permite al estudiante mostrar a sus compañeros su conocimiento, bien ganando o bien diseñando el juego, ya que ellos mismos pueden crear su propio juego y prepararlo fuera de clase.

Para ello les hice leer unos artículos relacionados con la unidad didáctica que estábamos tratando y les animé a diseñar preguntas para utilizar Kahoot. Un ejemplo que desarrollaron fue el siguiente:

1.- On what grounds is the natural approach based on?
A. Learning and acquisition
B. Theory of language
C. Theory of communication

2.- How do Terrel and Krashen understand language?
A. As a grammatical approach
B. As a vehicle for communicating meaning and messages
C. As a tool helping people understanding each other

3.- When does a teacher interact with a student in an oral way? 

A. After having listened to a story
B. While explaining new vocabulary
C. Only when the teacher thinks that the student is ready.

Al contrario de lo que comenta Tobías y otros (2014: 496) sobre el hecho de que los juegos especialmente diseñados con propósitos educativos no son tan divertidos como los diseñados específicamente para la diversión, cabe decir que la utilización de Kahoot en clase de la asignatura de didáctica de las lenguas extranjeras fue un éxito.

Se puede decir que Kahoot cumple con las características que destaca McClarty (en Walling 2014: 98) sobre los juegos digitales, es decir, están basados en principios de aprendizaje, involucran al alumno, ofrecen oportunidades personalizadas de aprendizaje, desarrollan habilidades del siglo XXI y ofrecen un ambiente de evaluación auténtico y real.

Podría añadirse que aquellos alumnos que sabían utilizar los dispositivos móviles con agilidad también los utilizaban mejor en el aprendizaje dentro y fuera del aula, como sistema de acceso a archivos, consultas a la plataforma o preparación de preguntas con Kahoot. También destaca el papel de aquellos alumnos digitalmente más competentes que en algunos casos ayudaron al resto de sus compañeros para acceder a este juego. En general, los alumnos consideraban que este tipo de herramientas multimedia iban a ser una realidad en el aula de enseñanza de Educación Primaria y que, en vez de libros de texto, encontrarían e-books, páginas web o tabletas para uso normal de los niños ${ }^{9}$.

Con respecto a los dispositivos móviles se puede añadir, como destaca Walling (2014: 29) centrándose especialmente en la tableta, que ofrecen nuevas oportunidades para el aprendizaje basado en la tecnología, ya que es básica para el uso de la Wiki o para jugar con Kahoot. Permite un aprendizaje más personalizado e individual que antes. Son los mismos alumnos los que diseñan los juegos con Kahoot o los que editan las

9 Por ejemplo, el programa Digital Text del Gobierno de la Rioja que ofreció a los centros educativos de La Rioja durante el curso 2013-14 la posibilidad de acceder a contenidos digitales, herramientas de gestión académica así como la creación, ordenación y personalización de libros y ejercicios digitales. Para ello los alumnos de primaria involucrados en dicho proyecto utilizaron libros de texto multimedia de Digital Text en la tableta Papyre Edu 1010 de Gammata, con sistema operativo Android y con procesador Intel, que ofrecía aplicaciones y accesorios para facilitar la investigación del alumnado de primaria. Para realizar el curso académico estos alumnos contaron con tablets, en vez de libros de texto y cuadernos para seguir las clases.

Para más información véase http://innovando.larioja.com/innovacion/240-libro-digitl.html 
páginas de la Wiki. Esto les permite de forma autónoma investigar, leer o ver videos relacionados con el tema a debate $y$, al tener que comentarlos, se hacen conscientes de sus deficiencias. Además, el profesor les puede hacer un seguimiento más cercano al comprobar sus aportaciones. Por otro lado, el alumno empieza a familiarizarse con las complejidades con las que se puede enfrentar en el mundo real ${ }^{10}$. En concreto, los alumnos de la asignatura de didáctica de las lenguas extranjeras pronto pusieron en práctica su conocimiento digital en la asignatura de Prácticum.

\section{El estudio}

En este estudio han participado un total de treinta y ocho estudiantes universitarios, de los que veintiuno era chicas y diecisiete, chicos. Casi todos ellos tenían entre veinte y veintiún años. Todos poseían ya un nivel B1 de inglés, siendo su lengua materna el español, a excepción de un alumno cuya lengua materna era el inglés. Esto les capacitaba para poder seguir las clases así como la lectura y visionado de los textos, ya que eran en inglés. Por otro lado, como ya se ha comentado, todos los alumnos tenían un dispositivo móvil del que poder hacer uso, por lo que la utilización de Wikis o Kahoot no representaba un problema técnico. Además prácticamente todos los alumnos eran digitalmente competentes, aunque si es cierto que poco a poco se fueron familiarizando cada vez más con estas dos herramientas. La herramienta de Kahoot les resultó a algunos alumnos un poco más complicado de manejar y también cabe mencionar que se vieron ayudados rápidamente por otros alumnos que supieron utilizarlo enseguida.

El instrumento de recolección de resultados de la aplicación de la clase inversa en la asignatura de didáctica de las lenguas extranjeras en el curso 2013-14 fue un cuestionario que cumplimentaron los alumnos y la observación directa. Casi al finalizar el curso se les hizo una encuesta para conocer su satisfacción con el modelo de la clase inversa, su acceso a los materiales y contenidos de aprendizaje de la asignatura así como su aprovechamiento en el aula y su participación en la misma. Por otro lado, la observación directa permitía valorar cómo los alumnos iban aprendiendo a utilizar mejor estas herramientas y a aprovechar las clases presenciales, como se apreciará a continuación.

10 Como sucede en el citado programa Digital Text. 


\section{Resultados}

Cuando se planteó el modelo de la clase inversa y la utilización de la Wiki y de Kahoot como forma de trabajar, los alumnos parecieron un poco desorientados, ya que su comportamiento en el aula como alumno cambiaba de forma notable. Les gustaba la idea de no tener que coger apuntes, pero daba la sensación de que no sabían muy bien qué se esperaba exactamente de ellos.

Al principio no estaban muy familiarizados con estas herramientas, pero aprendieron a manejarlas sin dificultad. Por ejemplo, una vez en la página de herramientas del aula virtual de la asignatura, fácilmente se introducían en la Wiki y podían introducir sus aportaciones o comentar las de otros compañeros. Apreciaban mejor que todos ellos eran parte del proceso de aprendizaje, su labor era muy activa, porque todos tenían que hacer sus resúmenes y comentarios para enriquecer la información final de cada tema, lo que permitía mucha agilidad.

La utilización de la Wiki permitía el acceso a la información a todos ellos en cualquier momento. Además como todos los alumnos leían cada entrada, ésto permitía que pudieran añadir información que considerasen pertinente o que hubiéramos destacado en clase durante esa semana y les fuera de especial relevancia. Esto podía provocar que algunas Wikis pudieran alargarse mucho. Por otro lado, esto permitía que todos los alumnos accedieran a la información de todos los textos, aunque personalmente solo hubiesen trabajado en uno por tema. Sin embargo, cabe decir que no fue hasta usar la Wiki varias veces cuando se sintieron cómodos manejando esta herramienta, compartiendo sus ideas, desarrollando tópicos y aceptando las sugerencias y comentarios de otros compañeros a sus ideas. Cabe añadir que con los textos que los alumnos tenían que trabajar en las Wikis, éstos trabajaban más el aspecto teórico en sus casas y las clases servían para centrarse más en sus dudas, en hacerles relacionar conceptos que apareciesen en distintos textos y temas y en hacerles apreciar más la importancia de lo que sucede en un aula de inglés en primaria, de cómo el profesor tiene que adaptar los conocimientos que tiene que impartir a sus alumnos de primaria y a sus estilos de aprendizaje.

Por otro lado, los alumnos universitarios se mostraron relativamente animados a utilizar Kahoot al principio, porque se trataba de un juego. Después de empezar a utilizar Kahoot se mostraron mucho más dispues- 
tos e involucrados en la preparación de las preguntas. Los alumnos se mostraron interesados y competitivos para utilizar sus conocimientos y ganar. Eso suponía aprender los contenidos, repasarlos o aprender de sus errores en un contexto lúdico. Ellos mismos diseñaban las preguntas y respuestas, lo que les permitía centrarse en aquellos aspectos que consideraban más importantes o que tuvieran mayor dificultad para ellos. De esta manera, también eran capaces de comprender cuál era su verdadero nivel de conocimiento sobre los temas que tenían que estudiar para el examen; además de suponer un repaso de los esos mismos temas.

Casi al finalizar el curso los alumnos reconocían que podían tener un acceso directo a los materiales y contenidos de aprendizaje de la asignatura. De hecho, los artículos o los vídeos los tenían que traer leídos o vistos antes de llegar a clase, a los que tenían acceso directo con la carpeta diseñada para tal motivo en el aula digital. Por otro lado, como en el aula virtual tenían mucha variedad de textos escritos y visuales, además de la ayuda bibliográfica que se les pudiera dar en horas de tutoría, el alumno podía elegir el tipo de materiales que se ajustasen mejor a su forma de aprender: textos escritos, textos visuales, vídeos, libros con actividades y ejemplos o Power Points ${ }^{11}$. Muchas veces el visionado de un vídeo, la lectura de ejemplos o los comentarios posteriores en el aula les hacían entender algún aspecto teórico y podía enriquecer los comentarios en el aula. Algunos alumnos venían con resúmenes, esquemas e, incluso, con la propuesta de visionar un video que habían encontrado en la web sobre el tema a tratar. La utilización de sus sugerencias les hacía sentirse más involucrados con la asignatura y facilitaba la interacción entre el alumno y profesor. El hecho de que luego se tuviera la clase, se comentaran las dudas sobre los textos y se expusieran ejemplos prácticos aunaba los ritmos de aprendizaje de la mayoría de los alumnos, porque en el aula se trataban una serie de aspectos de un tema y todos los alumnos tenían que reflexionar sobre esos aspectos. Además, esta forma de trabajo permitía al profesor centrarse más fácilmente en los puntos débiles. Esto requería más flexibilidad en el aula y una mayor propuesta de ejemplos por parte del profesor. Eran los propios alumnos los que de forma clara dictaban el ritmo de la clase, lo que hacía falta explicar o no. Además, por los comentarios de los alumnos se entendía que habían hecho sus

11 En la lista de herramientas de aprendizaje más útiles del Centre for Learning \& Performance Technologies (C4LPT) en el año 2014 aparece esta herramienta en la quinta posición. 
lecturas o visto los videos propuestos, al menos en su gran mayoría, por lo que prácticamente todos los alumnos se involucraron en este modelo de aprendizaje. Su participación y aprovechamiento en el aula era notable.

Cabe señalar que la información era compartida por todos antes de llegar a clase. Esto permitía clarificar las dudas más rápidamente y profundizar en aspectos que pudieran interesar más. Por tanto, las interacciones con el profesor eran prácticamente constantes y las clases se aprovechaban muy bien. Cuando un alumno planteaba una duda se veía ayudado por las intervenciones de sus compañeros. Esto daba lugar a una frecuencia de las interacciones entre los alumnos importante, especialmente a medida que iban adquiriendo experiencia con esta forma nueva de trabajo en clase. También hay que destacar que siempre había un pequeño grupo de alumnos que iniciaban y mantenían esta interacción. Sin embargo, se iba invitando a cada uno de los alumnos a participar para que todos se fueran sintiendo cómodos exponiendo sus ideas $y$, de alguna manera, todos tuvieran que desarrollar su pensamiento crítico. Por otro lado, se tenía en cuenta que los alumnos de un Grado de Educación acabarían dando clase en su futura carrera profesional, así que ésta era una buena manera de ayudarles a acostumbrarse a hablar delante de otras personas, a debatir, a mantener y defender su opinión o a cambiarla, si estaban equivocados.

\section{Conclusión}

Las nuevas tecnologías están cambiando la forma en que los docentes se enfrentan a su tarea en las aulas (Pilgrim, Bledsoe and Reily 2012: 16). Como se ha mostrado, este sistema de enseñanza de la clase inversa es una manera de involucrar al alumno, de interesarle por la asignatura y de facilitar la resolución de problemas y el desarrollo del pensamiento crítico, objetivos planteados con este modelo en la asignatura de didáctica de las lenguas extranjeras. Para ello los alumnos tuvieron que responsabilizarse de leer los textos o visionar los videos para poder participar en clase. Vieron, por ejemplo, cómo se cuenta un cuento y cómo ser consciente de la utilización del lenguaje corporal para añadir significado a lo que se dice en inglés. También comprobaron cómo es una clase con nuevas formas de enseñanza de inglés, como Jolly Phonics. La 
posibilidad de visionar los videos les dio tiempo a plantearse posibles respuestas a preguntas propuestas; lo que significaba comprender bien el texto para poder destacar los aspectos más relevantes y dar una buena respuesta. El papel del alumno pasaba a ser más activo que el que venía siendo el tradicional. No se esperaba solamente que el alumno diera respuesta a una serie de preguntas en un examen final. Con este sistema en la asignatura de didáctica de las lenguas extranjeras el alumno tenía que mostrarse activo en su proceso de aprendizaje de forma casi diaria, lo que también permitía una evaluación continua y formativa sobre el proceso de enseñanza y aprendizaje para mejorarlo. Por eso, se podría hablar de que el aprendizaje del alumno era más activo y experiencial (Cornelius 2008: 94), ya que la clase no se basaba en una lección magistral.

En este sentido se puede mencionar la pirámide del aprendizaje y el mayor porcentaje de retención después de 24 h con actividades relacionadas con prácticas y enseñanza a otros. Este tipo de aprendizaje también tiene que ver con el aprendizaje significativo, ya que el alumno relaciona la información que se le presenta con la que ya tiene y que le sirve de anclaje para poder comprender o reajustar su conocimiento. De hecho, los alumnos eran capaces de relacionar los contenidos de los nuevos textos con otros anteriores o con experiencias propias o cercanas a ellos.

En este ejemplo de clase inversa los estudiantes también desarrollaron un aprendizaje procedimental y práctico así como un pensamiento crítico y resolución de problemas. Por ejemplo, cuando tuvieron que comentar actividades de vocabulario que desarrollarían en una clase de inglés de Educación Primaria con diversos libros de textos, tipos de ejercicios y materiales. Así aprendieron a gestionar la información, a plantearse formas de resolver dudas sobre las actividades de vocabulario que tenían que comentar, a investigar y proponer soluciones para explicarlas mejor. Incluso sugirieron material añadido, como textos sobre la enseñanza y aprendizaje de vocabulario de inglés para justificar sus comentarios didácticos o videos con clases de inglés en Educación Primaria para ejemplificar sus respuestas. Estas actitudes les permitieron más fácilmente conseguir las competencias que se marcaban en la ficha de la asignatura. Además, practicaron el razonamiento y aplicaron el conocimiento en los comentarios didácticos de las actividades de vocabulario de inglés en Educación Primaria. También trabajaron en equipo 
y desarrollaron la comunicación oral, puesto que sus exposiciones en el aula eran orales, a veces en equipo y otras, individuales. Sin embargo, el trabajo en la Wiki siempre era en grupo, porque todos añadían información en las diferentes entradas de las Wikis. Por lo tanto, desarrollaron competencias que les ayudaban en su futura tarea profesional como docentes. Es decir, consiguieron otros dos de los objetivos trazados con el modelo de la enseñanza inversa, comunicación y colaboración así como habilidades para la vida y el trabajo.

Además, el hecho de que el centro de la clase no fuera el profesor, el hecho de que hubiera más participación por parte de los alumnos le permitió al profesor conocerlos mejor. Con todas las intervenciones que se dieron en clase el profesor supo entender mejor cuáles eran los puntos fuertes y débiles de sus alumnos así como se dio cuenta de qué temas les interesaban más. A su vez, el hecho de que los alumnos intervinieran más les permitió a ellos mismos conocerse mejor, darse cuenta de lo que entendían o de si se sabían expresar lo suficientemente bien como para explicar un tema. Hay que recordar que eran los alumnos los que comentaban y debatían, para ello tenían que saber decir y conocer el tema para explicarlo a los demás. Por tanto, casi cada comentario, cada debate, cada exposición daba la posibilidad al alumno a ser consciente de hasta dónde llegaba en la asignatura ese día, sin necesidad de una nota. Con este sistema de enseñanza el alumno era consciente de cómo mostraba lo aprendido tanto al profesor como al resto de sus compañeros. Para ello, cabe también destacar el hecho de que el ambiente en clase era de confianza y cooperación entre los alumnos. Eran alumnos adultos que estaban estudiando porque querían. Esto justificaría su interés por la asignatura, que era optativa, y ese ambiente de cooperación. Un sistema como este les permitía ser el centro de la clase; colaborar, investigar, tomar decisiones y relacionar su aprendizaje con las tecnologías de la información y comunicación. Además utilizaron estas tecnologías "como apoyo al aprendizaje colaborativo entre estudiantes, como ayuda a la adquisición de capacidades de aprendizaje autónomo y autorregulado, y como andamiaje a la resolución de problemas complejos enmarcados en tareas auténticas", aspectos que destaca Onrubia (2007: 21) al explicar las tecnologías de la información y la comunicación como instrumento de apoyo a la innovación de la docencia universitaria.

Como este sistema de enseñanza forma un tipo de alumno más activo, el profesor tiene que tener en cuenta las horas de clase y de estudio al 
día que tiene el alumno para que de una manera realista se le estimule a leer textos escritos, visionar un vídeo y ejercer su pensamiento crítico antes de Ilegar a clase. Además, también hay que tener en cuenta que el alumno tiene otras asignaturas con las que tiene que cumplir y el profesor debe ser consciente de este aspecto a la hora demandarles tareas para no sobrecargarles de trabajo para un mismo día. Por tanto, la coordinación entre los profesores es importante para que este sistema de enseñanza funcione mejor.

Merece la pena también destacar los comentarios de los alumnos cuando decían que eran clases más interactivas y que asimilaban mejor los contenidos. También resaltaron el uso de las Wikis, porque facilitaban el trabajo en grupo y permitía compartir el aprendizaje, y Kahoot, como un buen recurso para trabajar la evaluación y la asimilación de contenidos. Éstas eran unas herramientas nuevas a la hora de presentar sus trabajos. También pudieron utilizar su inventiva para introducirlos, de ahí la utilización también de Mind mapping y Popplet, y el diseño de diversos juegos basados en los contenidos de la materia en Kahoot. Se convirtieron en diseñadores y productores de contenidos digitales, aspecto que destaca Onrubia (2007: 33) al hablar de los alumnos universitarios como usuarios de las tecnologías de la información y de la comunicación en educación. Así en esta experiencia los alumnos pudieron conseguir otros dos objetivos marcados con el modelo de la clase inversa en la asignatura de didáctica de las lenguas extranjeras, creatividad e imaginación y desarrollo de la competencia tecnológica.

Bocconi, Panagiotis y Yves (2012: 8) señalan que una forma innovadora de práctica pedagógica es la que utiliza las nuevas tecnologías que facilita que el alumno sea el centro del proceso de aprendizaje, que facilita el trabajo en grupo, el aprendizaje significativo, el aprendizaje por medio de hacer, la resolución de problemas, la creatividad y el pensamiento crítico. Precisamente estos aspectos son los que se han conseguido en esta experiencia de "The Flipped Classroom" en la asignatura de didáctica de las lenguas extranjeras en un ambiente de implicación, interés y motivación entre los alumnos. 
Experiencia de la clase inversa en didáctica de las lenguas extranjeras

\section{Referencias bibliográficas}

Barcena, E., Read, T. y Arús, J. (Eds.). (2014). Language for specific purposes in the digital era. Nueva York: Springer.

Bocconi, S., Panagiotis, G. y Yves, P. (2012). JCR Scientic and policy reports. Innovating Learning: Key elements for developing creative classrooms in Europe. Luxemburgo: Publications office of the European Union.

Breeze, R. (2014). Moodle glossary tasks for teaching legal English. En Bárcena, Read y Arús (Eds.), Langugae for specific purposes in the digital era. (pp 111-129). Nueva York: Springer.

Cornelius, S. y Gordon, C. (2008). Providing a flexible, learner-centered programme: Challenges for educators. Internet and higher education, (11), 33-41.

De Miguel Díaz, M. (2006). Metodologías para optimizar el aprendizaje. Revista interuniversitaria de profesorado, 20 (3), 71-91.

Enfield, J. (2013). Looking at the impacto $f$ rthe flipped classroom model of instruction on undergraduate multimedia students at CSUN. TechTrends, (5), 14-22.

Hsu Y., Ching, Y. y Grabowski, B.L. (2014). Web 2.0 Applications and practices for learning thorugh collaboration. En Spector, Merril y Grabowski (Eds.), Handbook of research on educational communications and technology (pp 747-758). Nueva York: Springer.

Ibáñez, V.E. y Gómez Alemany, I. (2004) ¿Qué pasa cuando cooperamos? Hablan los alumnos. Investigación en la escuela, (54), 69-79.

Icard, B. (2014). Educational tecnologies best practices. International Journal of instructional technology and distance learning, 11, (3), 37-42.

Lot, C. 2005. Introduction to the Wiki. Distance Learning Systems. Center for Distance Education. Acceso 15 de mayo de 2010 en http://distance.uaf.edu/dls/resources/present/cc-aug-04/wiki/index.cfm

Mayer, R. (2014). Multimedia instruction. En Spector, Merril y Grabowski (Eds.), Handbook of research on educational communications and technology (pp 385-399). Nueva York: Springer.

Novak, J.D. y Cañas, A.J. (2006) The theory underlying concept maps and how to construct and use them. Technical Report IHMC C-map Tools. Florida Institute for Human and machine Cognition, Pensacola Fl. Acceso 1 de marzo de 2008 en http://cmap. ihmc.us/ Publications/ResearchPapers/TheoryCmaps/TheoryUnderlyingConceptMaps.htm

Marqués Graells, P. (2000) Impacto de la s TIC en la educación: funciones y limitaciones. 3 Ciencias. Revista de investigación. Acceso 3 de enro de 2014 en http:// www.3ciencias.com/wp-content/uploads/2013/01/impacto-de-las-tic.pdf

Onrubia, J. (2007). Las tecnologías de la información y la comunicación como instrumento de apoyo a la innovación de la docencia universitaria. Revista interuniversitaria de Formación de Profesorado, 21, (1), 21-37.

Pilgrim, J., Bledsoe, C. y Reily, S. New tecnologies in the classroom. The Delta Kappa Gamma bulletin, 78, (4), 16-22.

Randall, S. D., Dean, D.L. y Ball, N. (2013). Flipping the classroom and instructional 
technology integration in a college level information systems spreadsheet course. Educational technology development, (61), 563-580.

Ricoy, C., Sevillano, M. L., y Feliz, T. (2011). Competencias necesarias para la utilización de las principales herramientas de Internet en al educación. Revista de educación, (356), 483-507.

Rodríguez-Arancón, P. y Calle Martínez, C. (2014) A practical application of wikis for learning Business Englisg as a second language. En Bárcena, Read y Arús (Eds.), Langugae for specific purposes in the digital era (pp 155-173). Nueva York: Springer.

Spector, J.M., Merrill, M.D., Elen, J. y Bishop M.J. (eds) (2014). Handbook of research on educational communication and technology. Nueva York: Springer.

Tobías, S., Fletcher, J.D. y Wind A.P. Game-based learning. En Spector, Merril y Grabowski (Eds.), Handbook of research on educational communications and technology (pp 485-503). Nueva York: Springer.

Tourón, J., Santiago, R., y Diez, A. (2014). The Flipped Classroom: Cómo convertir la escuela en un espacio de aprendizaje. Méjico DF: Grupo Océano.

Trilling, B. y Fadel, C. (2009). 21st Century skills. Learning for life in for times. San Francisco: B Jossey-Bass.

Wald, M., LI, Y., Cockshull, G., Hulme, D., Moore, D., Purdy-Say, A., y Robinson, J. (2014). Synote second screening: using mobile devices for video annotation and control. ICCHP Proceedings, (14), 1-4.

Walling, R.D. (2014). Designing Learning for Tablet Classrooms. Innovations in Instruction. Nueva York: Springer.

Zabalza Berazana, M.A. (2006). La convergencia como oportunidad para mejorar la docencia universiratia. Revista interuniversitaria de profesorado, 20 (3), 37-69.

Zuraidah, S. (2014). The use of a flipped classroom to enhance engagement and promote active learning. Journal of education and practice, 5, (2), 124-131. 
